05

\title{
Электрокалорический эффект в твердом растворе $\mathrm{BaTiO}_{3}-\mathrm{SrTiO}_{3}$
}

(С Е.П. Смирнова, Г.Ю. Сотникова, Н.В. Зайцева, А.А. Капралов, Г.А. Гаврилов

Физико-технический институт им. А.Ф. Иофре РАН, Санкт-Петербург, Россия

E-mail: esmirnoffa@gmail.com

Поступило в Редакцию 17 августа 2017 г.

Проведены исследования электрокалорического эффекта, а также диэлектрических, пироэлектрических и пьезоэлектрических свойств твердого раствора $0.68 \mathrm{BaTiO}_{3}-0.32 \mathrm{SrTiO}_{3}$ в области фазового перехода первого рода в смещающем электрическом поле. Обсуждаются диэлектрический и электромеханический вклады в пироэлектрический и электрокалорические эффекты.

DOI: 10.21883/PJTF.2018.02.45464.17009

Электрокалорический эффект (ЕСЕ) - явление, при котором происходит обратимое изменение температуры материала при приложении электрического поля $[1,2]$. Создание охлаждающих устройств на основе электрокалорического эффекта рассматривается в настоящее время как один из возможных способов повышения их энергоэффективности и улучшения экологии окружающей среды за счет отказа от использования загрязняющих хладагентов [3]. Одним из направлений поиска новых материалов является исследование ЕCE при сегнетоэлектрических фазовых переходах [4]. Обратимое изменение температуры вещества $\delta T$ в результате электрокалорического эффекта при адиабатических условиях определяется соотношением [2]

$$
\delta T=-(T / \rho) \int\left(1 / C_{E}(T)\right)(\partial P(E) / \partial T)_{E} d E
$$

где $T-$ температура, $E-$ напряженность электрического поля, $P(E, T)$ - поляризация, $C_{E}(T)$ - удельная теплоемкость при постоянной напряженности, $\rho$ - плотность. Согласно уравнению $(1)$, величина $(\partial P(E) / \partial T)_{E}$ во многом определяет $\delta T$, и электрокалорический эффект 
при сегнетоэлектрических переходах может достигать максимальных величин. Известно, что при сегнетоэлектрических фазовых переходах первого рода поляризация изменяется скачкообразно, но при воздействии электрического поля „скачок“ размывается по температуре. Известно также, что пироэлектрический коэффициент $p$ материала определяется соотношением $p=(\partial P(E) / \partial T)_{E}$, а связь между пироэлектрическим $p$ и электрокалорическим коэффициентом $e$ дается выражением $e=-p T / C_{E}$. В свою очередь можно выделить два вклада в пироэффект. Это прежде всего диэлектрический вклад, он определяется изменением поляризации с температурой (первичный пироэффект). Кроме того, существенное влияние может оказывать электромеханическое взаимодействие, которое определяет вторичный пироэффект. Вклад вторичного пироэффекта в суммарный пироэлектрический коэффициент определяется соотношением $p^{\mathrm{II}}=d c \alpha$, где $d-$ пьезомодуль, $c-$ упругий модуль, $\alpha$ - коэффициент теплового расширения [1].

Пироэлектрический эффект при действии постоянного электрического поля изучался в сегнетоэлектриках как в области фазового перехода, так и вне перехода главным образом с точки зрения применимости материалов для работы в режиме диэлектрического болометра [5]. Особенности перехода практически не принимались во внимание. Исследование ЕСЕ, несмотря на возросший в последние годы интерес, в основном ограничивалось измерением $\delta T[3,4,6-8]$.

В то же время эффекты, возникающие при воздействии внешнего электрического поля, такие как диэлектрическая нелинейность, пьезоэффект, упругие свойства, тепловое расширение, различаются по полевым и температурным зависимостям. Целью настоящей работы является исследование ЕСЕ и сопутствующих эффектов, таких как пиро- и пьезоэффекты, а также температурное изменение упругих свойств при действии электрического поля в сегнетоэлектриках с фазовым переходом первого рода, для выявления механизмов, определяющих электрокалорический эффект в таких материалах.

В качестве объекта исследования был выбран твердый раствор титаната бария-стронция $0.68 \mathrm{BaTiO}_{3}-\mathrm{SrTiO}_{3}(\mathrm{BST})$, обладающий сегнетоэлектрическим переходом первого рода [9].

Керамические образцы BST были изготовлены по обычной технологии путем реакции в твердой фазе. Полнота образования твердых растворов контролировалась методом рентгеноструктурного анализа с использованием $\mathrm{Cu} K_{\alpha^{-}}$и $\mathrm{Co} K_{\alpha}$-излучения. Полученная керамика была

Письма в ЖТФ, 2018, том 44, вып. 2 
однофазной, обладала структурой перовскита и плотностью примерно 95\% от теоретической. Были проведены измерения температурных и полевых зависимостей диэлектрической проницаемости. Эффективный пьезомодуль $d^{e f f}$, зависящий от поля при данной температуре в области перехода, рассчитывался по формуле $S=d^{e f f} E$, где $S$ - деформация, возникающая при индуцированном электрическим полем пьезоэффекте в керамическом образце. Измерения продольной деформации проводились на гальваномагнитном дилатометре в квазистатическом режиме при температурах $15-45^{\circ} \mathrm{C}$.

Индуцированный постоянным электрическим полем пироэлектрический отклик в синтезированных образцах изучался с помощью динамического метода - метода модуляции интенсивности лазерного излучения (laser intensity modulation method - LIMM) [10]. Детальное описание измерений приводится в работе [11].

Электрокалорический эффект исследовался с помощью адиабатического калориметра, аналогичного прибору, описанному в [12]. Образцы для измерения представляли собой керамические диски диаметром $9 \mathrm{~mm}$ и толщиной $1 \mathrm{~mm}$ с электродами из возженного серебра. Все измерения проводились на механически свободных образцах.

Температурные зависимости диэлектрической проницаемости $\varepsilon$ для твердого раствора BST продемонстрировали существование сегнетоэлектрического фазового перехода из кубической в тетрагональную фазу с температурой максимума $T_{m}=25^{\circ} \mathrm{C}$ и $\varepsilon_{m}=16480$. При приложении постоянного смещающего электрического поля переход размывается с понижением максимальной величины диэлектрической проницаемости (рис. 1) и небольшим увеличением $T_{m}$ на $\sim 3^{\circ} \mathrm{C}$ при напряженности приложенного поля $15 \cdot 10^{5} \mathrm{~V} / \mathrm{m}$. На рис. 1 представлена также зависимость эффективного пьезомодуля $d^{e f f}(E)$ при температурах в области фазового перехода. Обращает на себя внимание тот факт, что при смещающем поле $E \approx 15 \cdot 10^{5} \mathrm{~V} / \mathrm{m}$ происходит насыщение зависимостей $\varepsilon(E)$ и $d^{e f f}(E)$. Температурные зависимости $\delta T$ (изменений температуры, возникающих в результате ЕСЕ) демонстрируют максимальные величины при напряженности электрического поля $15 \cdot 10^{5} \mathrm{~V} / \mathrm{m}$ и температуре на $4^{\circ} \mathrm{C}$ выше $T_{m}$ (рис. 2). Измеренная в работе максимальная величина $\delta T$ равняется $0.43^{\circ} \mathrm{C}$. Близкая величина $\delta T \approx 0.45^{\circ} \mathrm{C}$ при комнатной температуре была получена для многослойного конденсатора на основе $\mathrm{BaTiO}_{3}$, но при поле $E=300 \cdot 10^{5} \mathrm{~V} / \mathrm{m}[13]$.

4* Письма в ЖТФ, 2018, том 44, вып. 2 


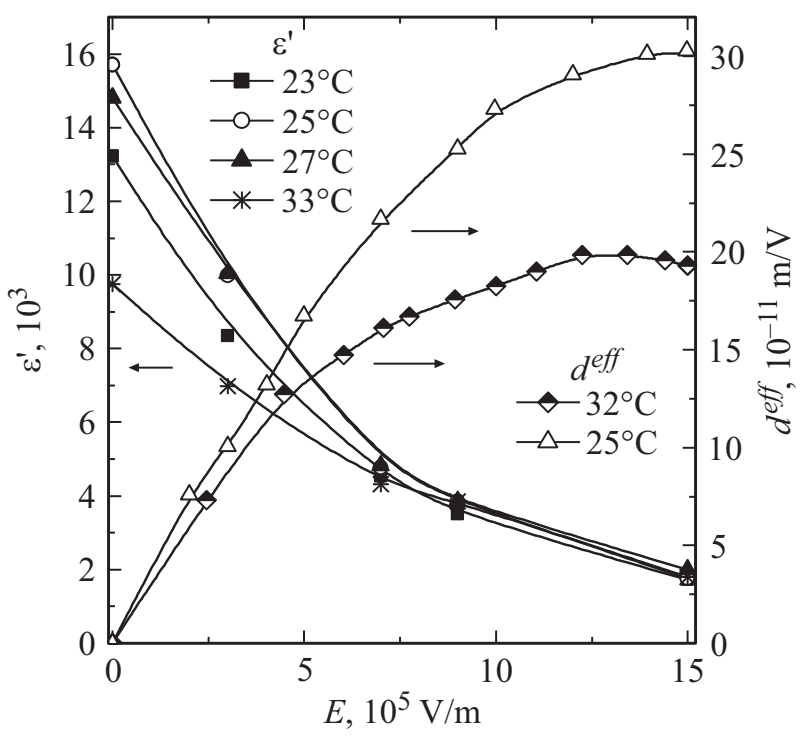

Рис. 1. Зависимость диэлектрической проницаемости $\varepsilon^{\prime}$ и эффективного пьезомодуля $d^{e f f}$ от величины приложенного поля для керамики BST при температурах, близких к $T_{m}$.

Особенностью фазового перехода первого рода является существование минимума (вплоть до отрицательных величин) коэффициента теплового расширения в области перехода, глубина которого уменьшается при приложении электрического поля [14]. Расчет температурной зависимости вторичного пирокоэффициента с учетом этого обстоятельства, а также температурной и полевой зависимостей $d^{e f f}$ (рис.1) и модуля упругости с [11] указывает на существование минимума $p^{\mathrm{II}}(T)$ при $T_{m}=25^{\circ} \mathrm{C}$ (рис. 3, $a$ ). Глубина минимума зависимости $p^{\mathrm{II}}(T)$ уменьшается с увеличением приложенного поля, но минимум сохраняется и при $E=15 \cdot 10^{5} \mathrm{~V} / \mathrm{m}$, при котором наблюдается максимальная величина $\delta T(T, E)$ (рис. 2 и $3, b)$. Влияние коэффициента теплового расширения определяет сдвиг максимума температурной зависимости измеренного пироэлектрического коэффициента в область более высоких температур, где $\alpha \geqslant 0$ (рис. 3,a). Максимальная величина $\delta T(T)$ при оптимальном приложенном поле $15 \cdot 10^{5} \mathrm{~V} / \mathrm{m}$ наблюдается при еще

Письма в ЖТФ, 2018, том 44, вып. 2 


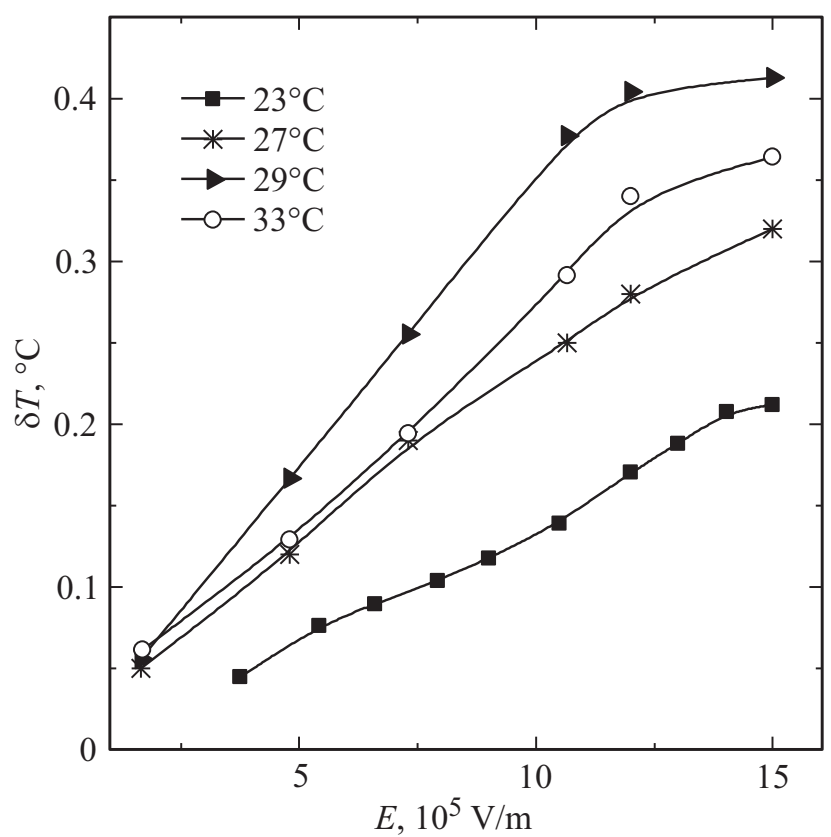

Рис. 2. Зависимость изменения температуры $\delta T$ в результате электрокалорического эффекта от электрического поля при температурах, близких к $T_{m}$.

более высокой температуре: $31^{\circ} \mathrm{C}$, что на $6^{\circ} \mathrm{C}$ выше $T_{m}$ (рис. $3, b$ ). Видно, что именно при этой температуре вторичный пирокоэффициент достигает максимальной величины $p^{\mathrm{II}}=2.63 \cdot 10^{-4} \mathrm{C} \cdot \mathrm{m}^{-2} \cdot \mathrm{K}^{-1}$ и составляет $18.8 \%$ от величины измеренного пирокоэффициента. Следует заметить, что исходя из соотношения для связи электрокалорического и пироэлектрического коэффициентов электромеханический вклад (вторичный пироэффект) в электрокалорический также составляет $18.8 \%$. Некоторый сдвиг температуры максимума $\delta T(T)$ относительно максимума $p(T)$ можно отнести к влиянию температурной и полевой зависимости теплоемкости. Это предположение нуждается в дополнительном изучении.

Таким образом, полученные результаты комплексного исследования позволяют проанализировать влияние различных механизмов, вносящих

Письма в ЖТФ, 2018, том 44, вып. 2 

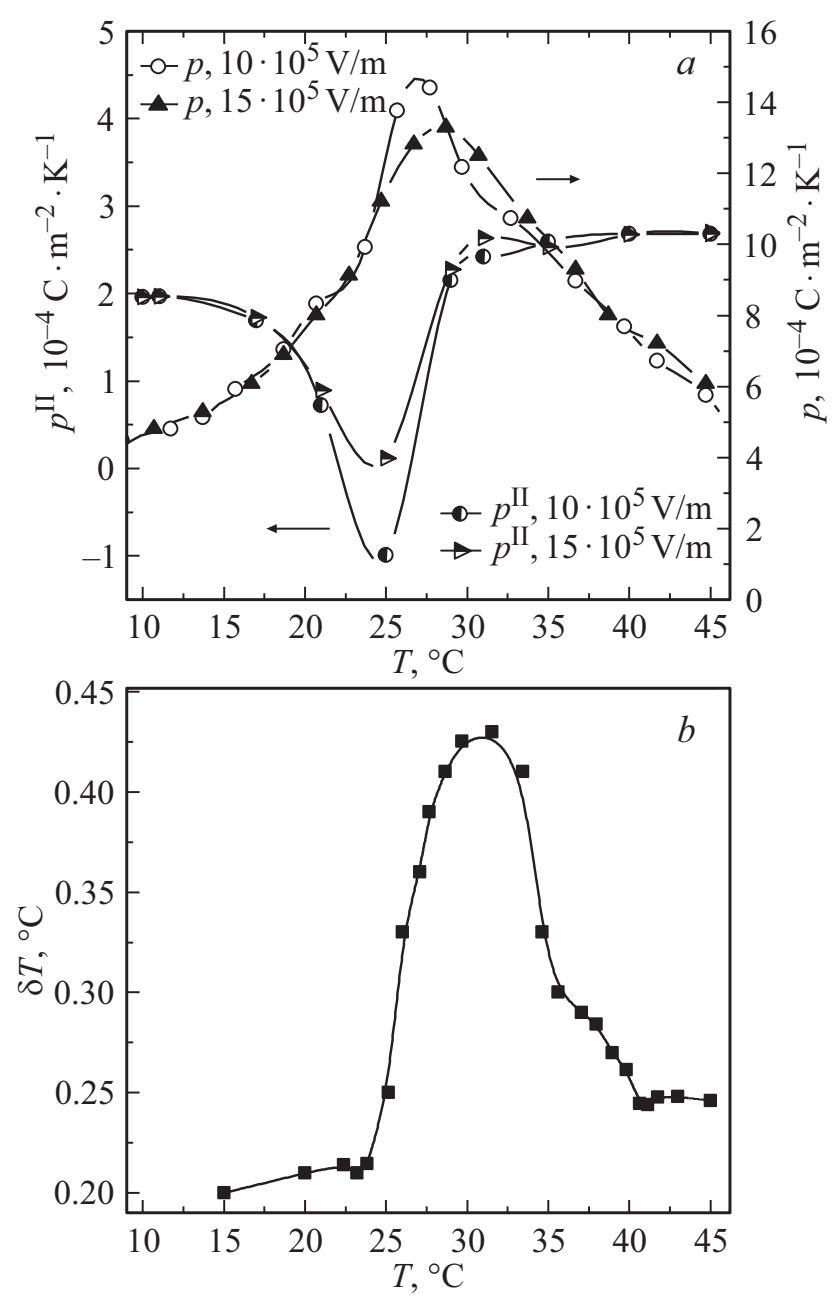

Рис. 3. Зависимости пироэлектрического коэффициента $p$ и коэффициента вторичного пироэффекта $p^{\mathrm{II}}$ от температуры при напряженности электрического поля $10 \cdot 10^{5}$ и $15 \cdot 10^{5} \mathrm{~V} / \mathrm{m}(a)$, зависимость $\delta T$ от температуры при оптимальном приложенном поле $15 \cdot 10^{5} \mathrm{~V} / \mathrm{m}(b)$. 
вклад как в пироэлектрический, так и в электрокалорический эффект в сегнетоэлектрике с фазовым переходом первого рода, и оценить роль вторичного пироэффекта в электрокалорическом эффекте.

\section{Список литературы}

[1] Най Джс. // Физические свойства кристаллов. М.: Мир, 1967. 385 с.

[2] Lawless W.N. // Phys. Rev. B. 1977. V. 16. P. 433-439.

[3] Crossley S., Mathur N.D., Moya X. // AIP Adv. 2015. V. 5. P. 067153 (1-8).

[4] Valant M. // Prog. Mater. Sci. 2012. V. 57. P. 980-1009.

[5] Whatmore R.W., Watton R. // Infrared detectors and emitters: materials and devices / Eds P. Capper, C.T. Elliott. Electronic Mater. Ser., Boston: Springer, 2001. V. 8. P. $99-147$.

[6] Electrocaloric materials: new generation of cooler. / Eds T. Correia, Q. Zhang. Berlin-Heidelberg-London: Springer-Verlag, 2014. $253 \mathrm{p}$.

[7] Scott J.F. // Ann. Rev. Mater. Res. 2011. V. 41. P. 229-240.

[8] Moya X., Kar-Narayan S., Mathur N.D. // Nature Mater. 2014. V. 13. P. 439450.

[9] Lemanov V.V., Smirnova E.P., Sirnikov P.P., Tarakanov E.A // Phys. Rev. B. 1996. V. 52. P. $3151-3157$.

[10] Lang S.B., Das-Gupta D.K. // Ferroelectrics. 1981. V. 39. P. 1249-1252.

[11] Смирнова Е.П., Сотников А.В. // ФТТ. 2006. Т. 48. В. 1. С. 95-98.

[12] Shebanovs L., Borman K., Lawless W.N., Kalvane A. // Ferroelectrics. 2002. V. 273. P. 137-142.

[13] Kar-Narayan S., Mathur N.D. // J. Phys. D: Appl. Phys. 2010. V. 43. P. 032002.

[14] Борман К.А., Фрицберг В.Я. // Фазовые переходы в сегнетоэлектриках. Рига: Зинатне, 1971. С. 155.

Письма в ЖТФ, 2018, том 44, вып. 2 